\title{
Research Status of Wind Energy Piezoelectric Generator
}

\author{
Chunhua Sun*, Guangqing Shang \\ Department of Mechanic and Electronic Engineering, Suzhou Vocational University, Suzhou, China \\ Email: *chh_sunny@163.com
}

How to cite this paper: Sun, C.H. and Shang, G.Q. (2018) Research Status of Wind Energy Piezoelectric Generator. Energy and Power Engineering, 10, 487-507. https://doi.org/10.4236/epe.2018.1012031

Received: November 6, 2018

Accepted: December 18, 2018

Published: December 21, 2018

Copyright $\odot 2018$ by authors and Scientific Research Publishing Inc. This work is licensed under the Creative Commons Attribution-NonCommercial International License (CC BY-NC 4.0). http://creativecommons.org/licenses/by-nc/4.0/

\begin{abstract}
It is of great significance for developing self-powered micro-devices to explore the research of piezoelectric effect in conversion of wind energy into electricity. Based on the different excitation modes, the existing wind energy piezoelectric generators are firstly classified. The research status of wind piezoelectric generators is further analyzed, and characteristics of various types of wind energy piezoelectric generators are summarized. Finally, the future research direction and emphasis of wind energy piezoelectric generators is proposed to carry out its miniaturization, lightweight and integration.
\end{abstract}

\section{Keywords}

Wind Energy, Piezoelectric Effect, Micro Generator

\section{Introduction}

With the development of low power products such as wireless sensor networks and MEMS, etc., higher requirements for power supply life, volume and maintenance cost are put forward. However, the traditional chemical batteries have some problems, such as limited energy density, high cost, periodic replacement, easily caused environmental pollution and so on. Exploring new power supply mode is an urgent problem for developing micro device [1].

Among all kinds of green energy sources in nature, wind energy has attracted much more attention for its abundant, clean, safe and wide distribution. The conversion modes of wind energy are mainly electromagnetic, electrostatic and piezoelectric [2]. Among these, the piezoelectric devices have become one focus in developing micro-devices in that its simple structure, small size, pollution-free, high energy density and so on. The paper will describe the research status of wind energy piezoelectric generator for furtherly understanding characteristics of the wind energy piezoelectric generators. 


\section{On Wind Energy Piezoelectric Generator}

According to the different excitation modes, wind piezoelectric generator can be divided into the following types, including windmill, vortex-induced, galloping motion, directly excited, resonant cavity and hybrid.

\subsection{The Windmill Type}

Windmill piezoelectric generator uses wind to drive the blade or turbine to rotate and to excite piezoelectric vibrator's motion for generating power through indirect excitation components. The indirect excitation components may be a pick, convex tooth, steel ball, or magnet mass. When using a magnet mass as a exciter, the type is called a non-contact excitation.

Priya designed a windmill type piezoelectric generator at 2005, as shown in Figure 1 [3]. The twelve piezoelectric bimorph beams were evenly arranged around the cylindrical pipe around the horizontal windmill axis, and a moving plate was also equipped on the axis. When the generator was working, the blade was driven to rotate by the wind and the generated torque was transmitted to the windmill axis through the cam mechanism and the suspended mass to drive the pick and the piezoelectric bimorph beams to vibrate and generate electric charge. When matching resistance and wind speed were $6.7 \mathrm{k} \Omega$ and $4.47 \mathrm{~m} / \mathrm{s}$ respectively, $7.5 \mathrm{~mW}$ electrical energy was generated which could meet the energy supply of microelectronic sensors. The energy conversion efficiency of the generator was $18 \%$, while one of the other same generators was only $1 \%$.

In order to improve the capturing efficiency of wind energy, Myers et al. [4] proposed a windmill piezoelectric generator with three blades at 2007, as shown in Figure 2. The internal crank mechanism of the generator transformed the vertical rotation into horizontal motion to impact two groups of piezoelectric vibrators to do vibration and generate electricity. One group of piezoelectric vibrator was composed of 9 piezoelectric bimorph beams. The entire generator was carved out of ABS plastic. The overall structure size of the generator was $76.2 \mathrm{~mm} \times 101.6 \mathrm{~mm} \times 127 \mathrm{~mm}$. When the average wind speed reached $2.8 \mathrm{~m} / \mathrm{s}$,

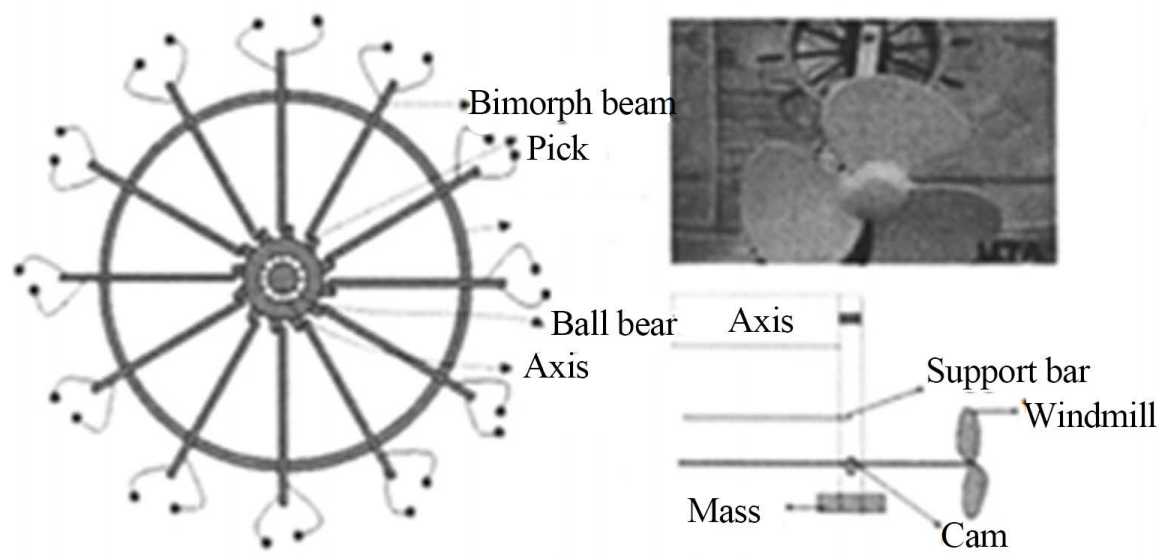

Figure 1. Windmill piezoelectric generator by Priya [3]. 


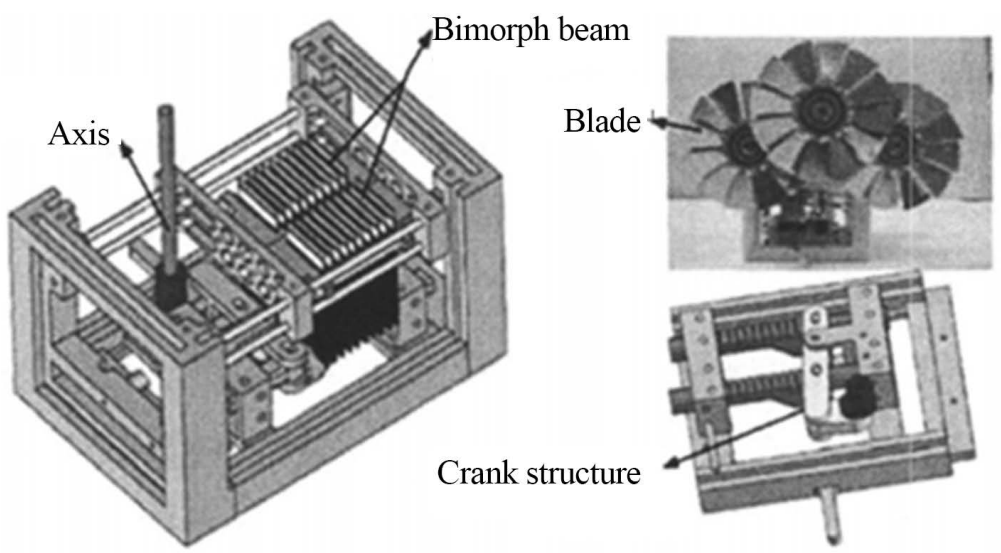

Figure 2. Piezoelectric generator with three blades by Myers et al. [4].

the power generation device could generate nearly $5 \mathrm{~mW}$ of electrical energy. This generator improved the capturing and conversion efficiency, the total working efficiency increased much more than the one in Ref [3].

Based on the systematic analysis of the basic mechanism of wind energy piezoelectric power generation, Zhang [5] proposed two design schemes, as shown in Figure 3. The first one used the wheel for capturing the wind energy to rotate and drive the horizontal axis and the hub, so that the free-moving steel ball in the hub impacted the free end of the cantilever to produce vibration, thus converting the wind energy into electricity. The 2nd transferred the wind wheel's horizontal rotation into linear motion of the cam-worm mechanism, thereby driving the motion of the cantilever piezoelectric vibrator on the beam. The experimental results showed that the power generation efficiency of the 2nd generator was higher, and its structure was simpler and the wind speed range was wider than the 1st one. But this generator's size was too larger to use in micro-device.

An impact type piezoelectric windmill generator was proposed by Yang at 2014, as shown in Figure 4 [6]. When the generator was working, the piezoelectric bimorph beams was struck by the free-moving steel ball and generated electricity. When 10 piezoelectric bimorph beams and 7 steel balls were used, 613 $\mathrm{uW}$ of electricity can be output at the speed of $200 \mathrm{r} / \mathrm{min}$ and matching resistance of $20 \mathrm{~K} \Omega$. The structure was simpler than the above ones. The impact vibration was also used to trigger the free movement of bimorph beams and generate much more electricity.

With the study in a deep-going way, many researchers not only improved and created the structure, but also studied the mechanism of wind-induced piezoelectric power generation. Based on the mathematical modeling and FEM analysis, Liang [7] concluded that the triangular piezoelectric cantilever with inner arc had better output characteristics. An impact type of PVDF piezoelectric harvester with wind direction restraint device was designed and manufactured, as shown in Figure 5. The experimental results showed that the optimized device reduced the starting wind speed from $4 \mathrm{~m} / \mathrm{s}$ to $2 \mathrm{~m} / \mathrm{s}$, and the maximum output 


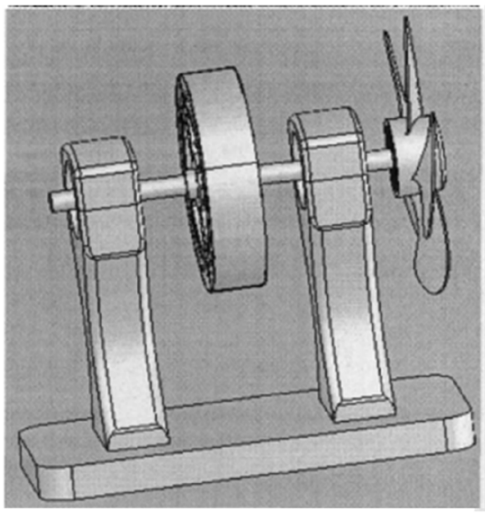

(a)

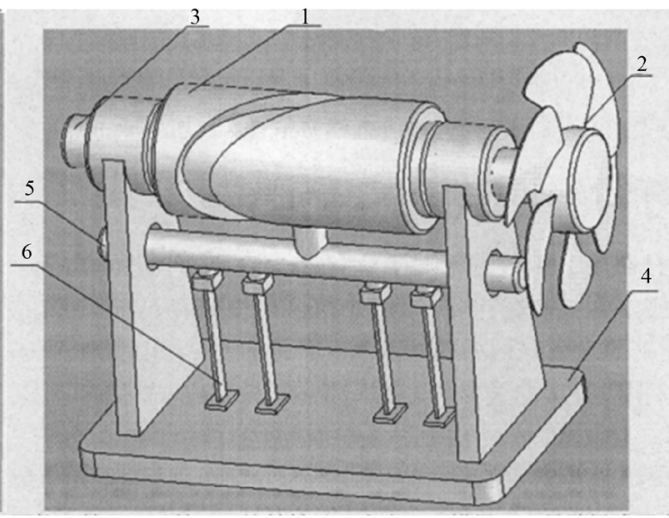

(b)

Figure 3. The schemes of two kinds of wind generators by Zhang [5]. (a) The first one; (b) The second one.

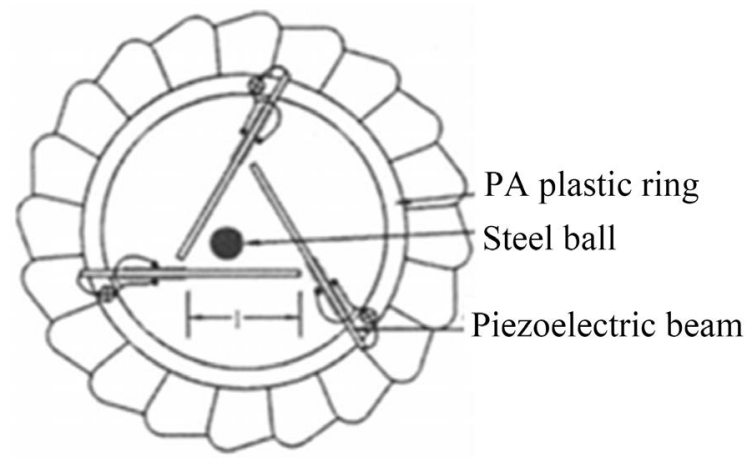

Figure 4. An impact type piezoelectric windmill generator by Yang [6].

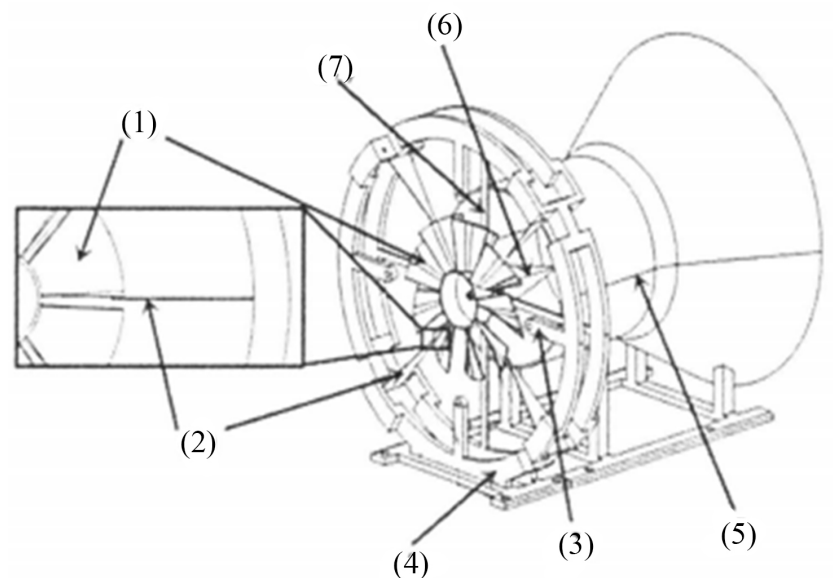

Figure 5. An impact type piezoelectric windmill generator with restraint device by Liang [7].

power was up to $198 \mathrm{uW}$. If two cantilevers were connected in parallel, the output power was increased to $282 \mathrm{uW}$.

$\mathrm{Xu}$ [8] and Luo [9] were proposed the piezoelectric generator with wind cups to improve capturing efficiency of wind energy at 2017-2018, as shown in Figure 6. While the generator was working, the stick rotated with the wind cups to make 


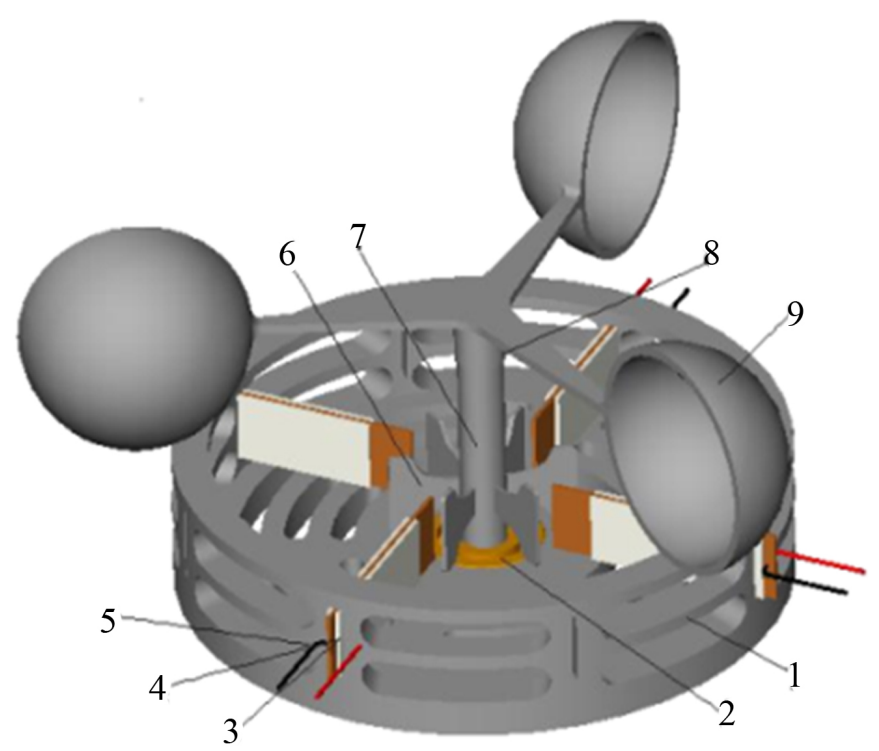

Figure 6. The piezoelectric generator with wind cups by Xu and Luo [8] [9].

the piezoelectric vibrators excite by themselves. The results showed that the starting wind speed was about $3 \mathrm{~m} / \mathrm{s}$, and the optimal load of rectangular or trapezoidal piezoelectric cantilevers was $25 \mathrm{~K} \Omega$ with corresponding output power of $0.11 \mathrm{~mW}$ and $0.13 \mathrm{~mW}$, respectively. Meanwhile, the optimum load of triangular piezoelectric cantilever was $20 \mathrm{~K} \Omega$, and the corresponding output power was $0.18 \mathrm{~mW}$.

In order to reduce friction loss and prolong service life of the generators, many scholars had devoted themselves to the development of contactless piezoelectric devices. A windmill generator using magnetic force to make piezoelectric cantilever vibrate was designed by Karami at 2013, as shown in Figure 7 [10]. The size of PZT-5A piezoelectric bimorph cantilever was $58 \mathrm{~mm} \times 12.7$ $\mathrm{mm} \times 0.38 \mathrm{~mm}$, and the overall size of the device was $80 \mathrm{~mm} \times 80 \mathrm{~mm} \times 175$ $\mathrm{mm}$. The permanent magnets with the same polarity were mounted at the windmill and the free end of the piezoelectric bimorph beams. When the windmill was rotating, periodic reciprocating vibration of piezoelectric bimorph beam occurred under the combined action of magnetic force and mechanical restoring force that made the piezoelectric beam generate electric energy. The axial and tangential arrangement of magnets was studied in the experiment. The experimental results showed that the tangential arrangement was better and generated several milliwatts of electricity at the wind speed of $2 \mathrm{~m} / \mathrm{s}$.

To meet the development of micro-device, the micro windmill generator was designed by Nasrin at 2015, as shown in Figure 8 [11]. Under the action of the wind, the blades rotated with the hub and the magnets. Due to the interaction among magnets, the piezoelectric cantilever produced periodic up-down vibration, and finally converted wind into electricity. The starting speed of the generator was $2.1 \mathrm{~m} / \mathrm{s}$, and the maximum output power density was $0.59 \mathrm{~mW} / \mathrm{cm}^{3}$.

Figure 9 showed the piezoelectric generator proposed by Ji and et al. at 2016 


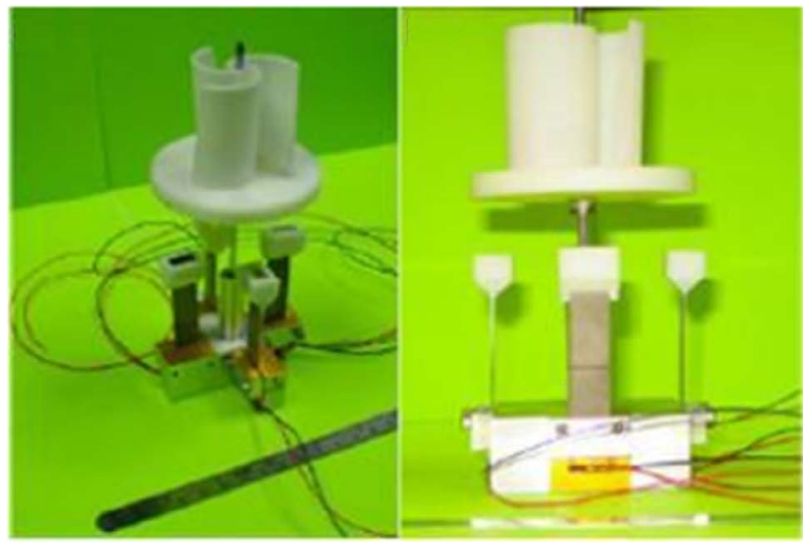

(a)

(b)

Figure 7. Magnetic type of windmill piezoelectric generator by Karami [10].

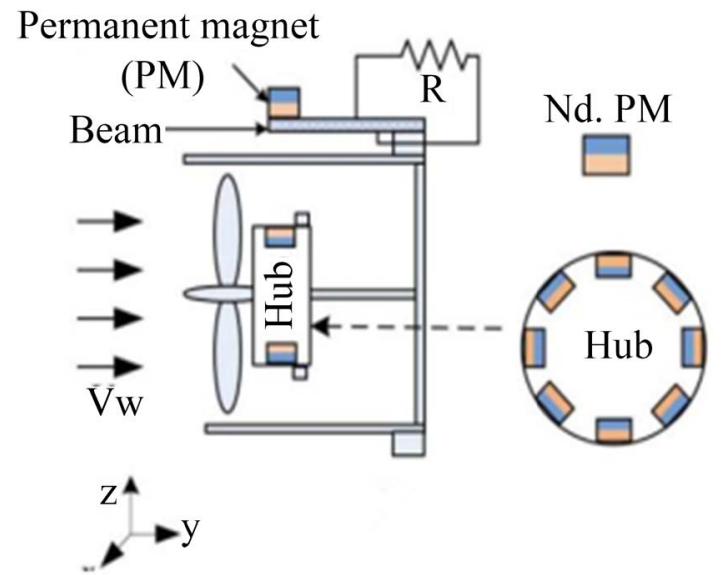

Figure 8. Micro windmill generator with magnetic force by Nasrin and et al. [11].

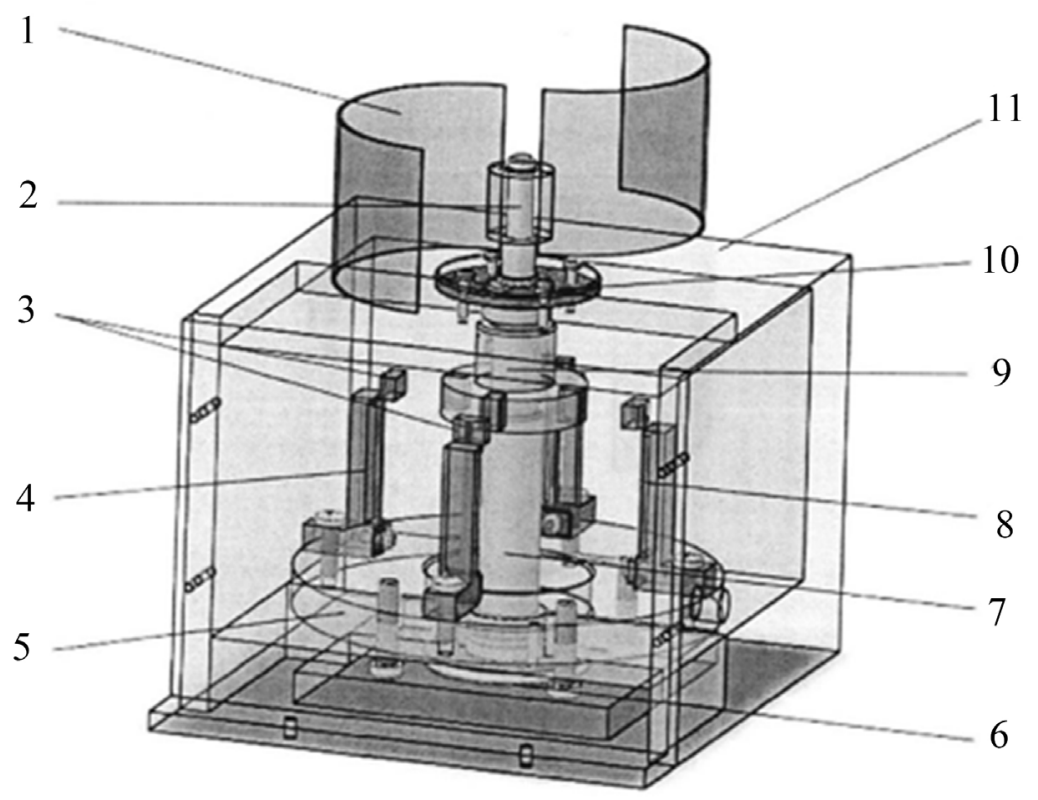

Figure 9. The piezoelectric generator with magnetic force by Ji et al. [12]. 
[12]. In order to reduce the starting wind speed, the generator adopted vertical axis and $S$ type impeller. Magnets were installed on the rotating shaft and cantilever beams. Meanwhile, to increase the working bandwidth and collect more wind energy, a block or arc-shaped frequency-increasing buffers were added into the generator. Thus, the working wind speed range increased from $5.38 \mathrm{~m} / \mathrm{s}$ $6.38 \mathrm{~m} / \mathrm{s}$ to $2.92 \mathrm{~m} / \mathrm{s}-13.0 \mathrm{~m} / \mathrm{s}$ and the working frequency bandwidth increased by 9.1 times, and the optimum matching resistance decreased from $350 \mathrm{k} \Omega$ to 1 $\mathrm{k} \Omega$.

\subsection{Vortex-Induced Type}

Wind energy harvesting piezoelectric device based on vortex-induced vibration generates electricity through a piezoelectric oscillator. The vortex-induced part may be a kind of blunt structure such as cylinder or block. It is set at the front of the piezoelectric oscillator along the wind direction. Karman whirlpool is formed to drive the oscillator for vibrating when the generator is working. Based on the working principle, Akaydin et al. [13] studied the energy harvesting efficiency in unstable whirlpool and analyzed the energy harvesting of the beam at different positions in the fluid. They found that the energy harvesting efficiency was only $0.0035 \%$. To improve the efficiency, Weinstein and et al. [14] put forward a power generating device with fins on piezoelectric cantilever at 2012, as shown in Figure 10. In the range of $2-5 \mathrm{~m} / \mathrm{s}$ wind speed, the additional fin could adapt to the external frequency and achieved resonance, thus improving the power generation efficiency. The generator generated $200 \mathrm{uW}$ power in the diameter of $15 \mathrm{~cm}$ pipe when the average wind speed was $2.5 \mathrm{~m} / \mathrm{s}$. When the flow rate increased to $5 \mathrm{~m} / \mathrm{s}, 3 \mathrm{~mW}$ of the electricity could generate.

In 2013, Sun and et al. [15] proposed a vortex-induced generator with a small wing at the free end of a piezoelectric beam, as shown in Figure 11 . When the wind passed through the wing, periodic lift and drag on the upper and lower sides was triggered. Because of the existence of blunt body, the lift force of wind increased, which induced the cantilever beam to bend upward and periodically swing under the action of inertia force and mechanical restoring force, thus converting wind energy into electric energy. The device was fabricated by thick

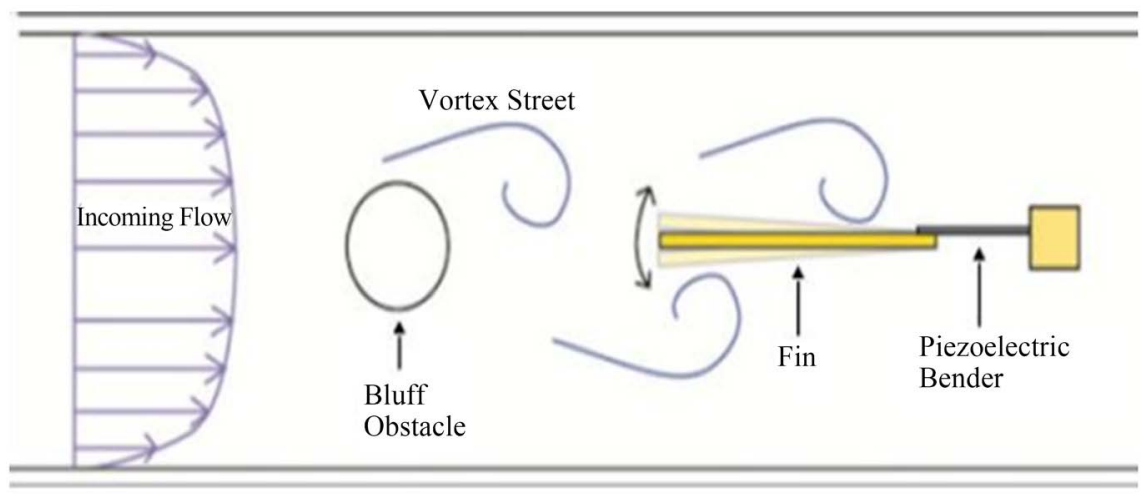

Figure 10. Wind energy generator with fins by Weinstein et al. [14]. 


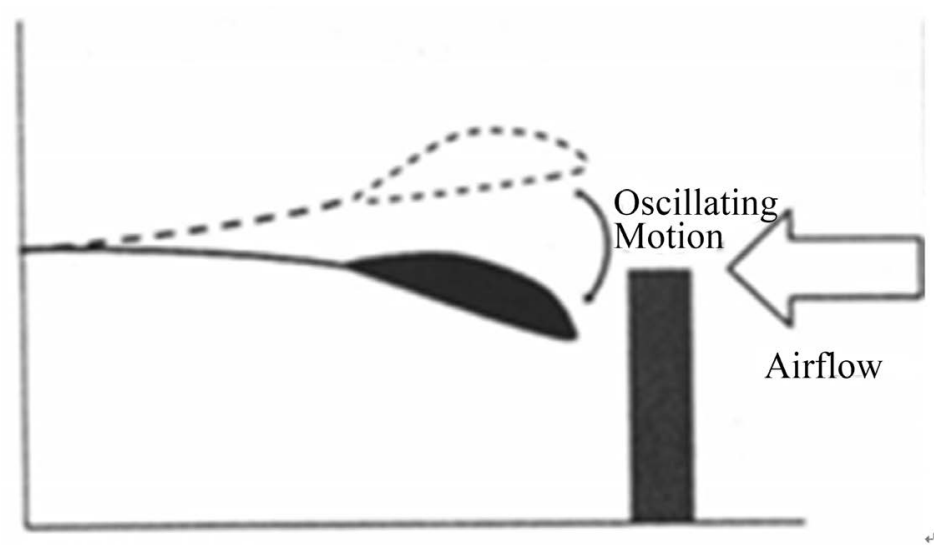

Figure 11. A vortex-induced generator with a small wing by Sun et al. [15].

film screen printing technology. Its overall size was $37.5 \mathrm{~mm} \times 37.5 \mathrm{~mm} \times 37.5$ $\mathrm{mm}$. It generated $0.1-0.85 \mathrm{uW}$ power and $0.5-1.32 \mathrm{~V}$ open circuit voltage in the range of $1.5-8 \mathrm{~m} / \mathrm{s}$ wind speed.

He [16] designed a MEMS piezoelectric generator based on vortex-induced and micro impact in 2013, as shown in Figure 12. The flexible cantilever beam had a certain angle with the direction of wind speed to increase the harvesting energy area. When the wind speed exceeded the critical one of the flexible cantilever beam, it produced violent vibration and caused periodic continuous collision with the colliding body. Thus, under the combined effect of vortex-induced and collision, the device gained more energy than the only vortex-induced generator. When the wind speed was $15.9 \mathrm{~m} / \mathrm{s}$ and the resistance of $100 \mathrm{k} \Omega$ was connected, the effective output voltage of the generator was $406 \mathrm{mV}$ and the corresponding output power was $1.6 \mathrm{uW}$.

\subsection{Galloping Motion Type}

Wind energy harvesting piezoelectric device based on galloping motion adopts to connect the bluff body directly with the piezoelectric vibrator. When the bluff body swings under the action of wind, it takes vibration with the piezoelectric vibrator to generate electricity. In 2011, Sirohi [17] designed a mirco galloping motion piezoelectric generator, as shown in Figure 13. The generator was mainly composed of two piezoelectric cantilevers and one equilateral triangular prism. The two piezoelectric cantilevers were attached to the two ends of the prism respectively which size was $72.4 \mathrm{~mm} \times 36.2 \mathrm{~mm} \times 0.267 \mathrm{~mm}$. The prism as a bluff body generated galloping motion under the action of wind, and thus driving the piezoelectric cantilevers to vibrate. The test results showed that when the wind speed was $11.6 \mathrm{mph}$, the maximum output power was up to $53 \mathrm{~mW}$.

In 2016, $\mathrm{Hu}$ [18] designed a miniature wind power generator based on galloping motion with one square prism shown in Figure 14. The generator added PET slices on the edges of the prism. Compared with the generator without PET slices on the prism, the output power of the proposed generator increased by about $150 \%$ when the wind speed was $2 \mathrm{~m} / \mathrm{s}$. 


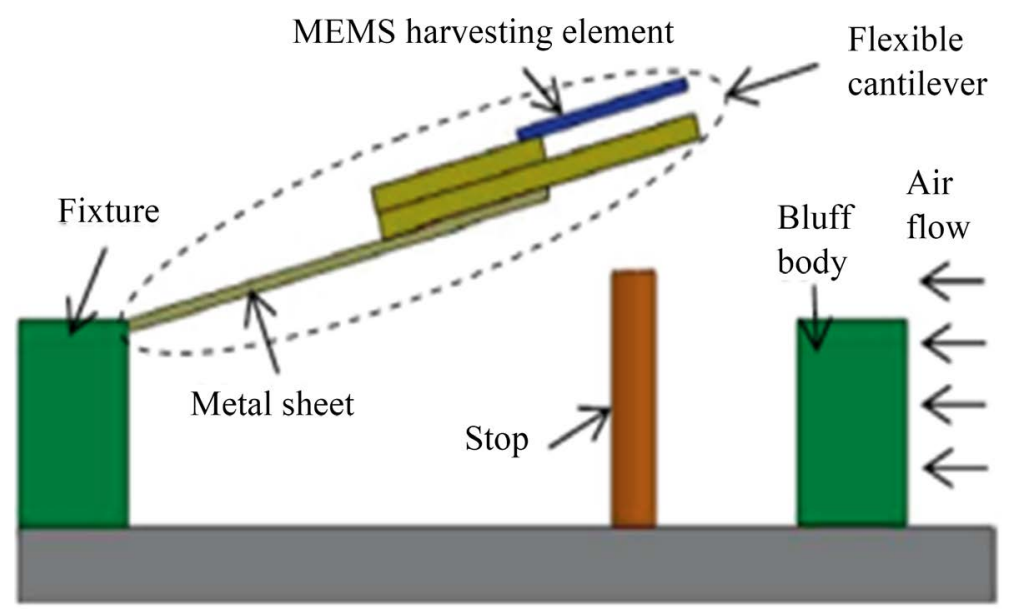

Figure 12. MEMS piezoelectric generator based on vortex-induced and micro impact [16].

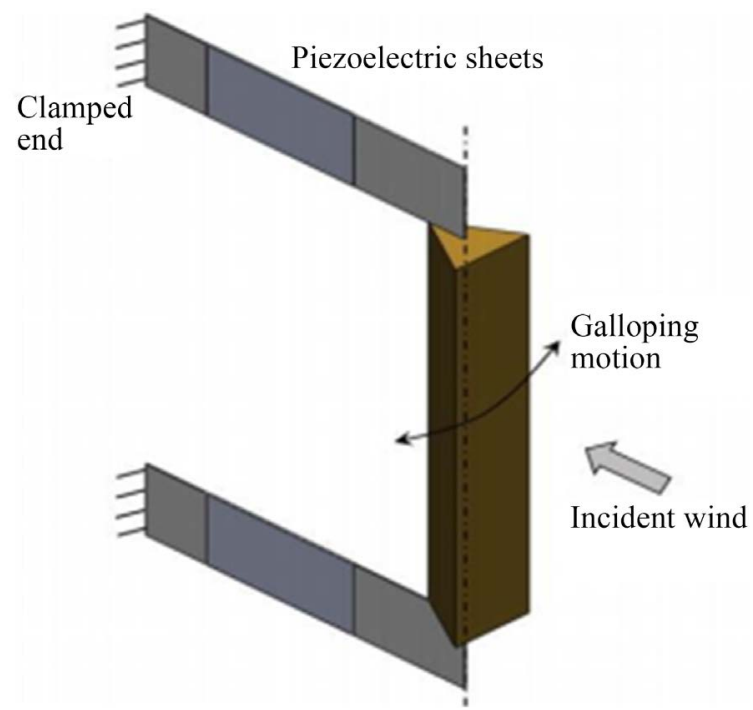

Figure 13. Mirco galloping motion piezoelectric generator by Sirohi [17].

Square prism

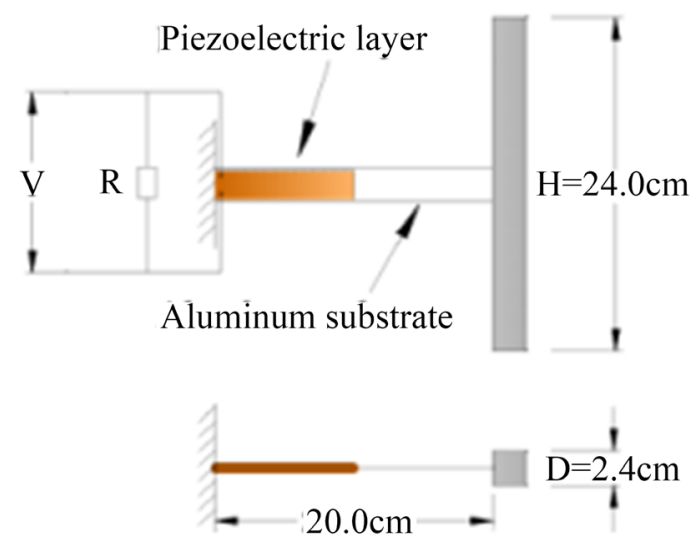

Figure 14. A miniature wind power generator based on galloping motion with four prism by $\mathrm{Hu}[18]$. 


\subsection{Directly Excited Type}

The directly excited piezoelectric generator generates the Karmen whirlpool on the rear side of the beam through the blast of the overpressure electric beam. The alternately shedding of the whirlpool changes the front and rear side pressure on the vibrator alternately, so that the piezoelectric beam makes self-excited vibration to generate electricity.

The typical directly excited piezoelectric generator is the piezoelectric bionic tree. Figure 15 showed the device proposed by Li and et al. [19]. The generator had 5 vibrating units which were called "leaves". Each "leaf" consisted of PVDF "stem", plastic hinge and isosceles triangular polymer plastic "blade". One end of PVDF stem was fixed on the bracket, which was perpendicular to the direction of airflow. The free end of the stem and the blade were connected through the plastic hinge plate to form an " $\mathrm{L}$ " shape. When a single vertical stem structure was adopted, the critical starting wind speed was $3.5 \mathrm{~m} / \mathrm{s}$. The maximum output power was $296 \mathrm{uW}$ when the wind speed was $8 \mathrm{~m} / \mathrm{s}$ and the external load $10 \mathrm{M} \Omega$.

In order to increase the power density, the effect of "blade" and "stem" on the output performance was further studied in Ref. [20]. The test results showed that the vertical arrangement structure was more suitable for capturing wind and its amplitude was larger than that of the parallel arrangement. The critical starting wind speed and the maximum output power of the generator increased with the area of equilateral triangle. When the "stem" was a narrow PVDF with a size of $41 \mathrm{~mm} \times 8 \mathrm{~mm} \times 0.205 \mathrm{~mm}$ and loaded with $30 \mathrm{M} \Omega$, the maximum output power of $0.14 \mathrm{~mW}$ was obtained under the wind speed of $7 \mathrm{~m} / \mathrm{s}$. At this time, the maximum power density was $2.036 \mathrm{~mW} / \mathrm{cm}^{3}$. When the stem was a double-layer PVDF with a size of $72 \mathrm{~mm} \times 16 \mathrm{~mm} \times 0.41 \mathrm{~mm}$, the maximum output power is $0.615 \mathrm{~mW}$ at wind speed of $8 \mathrm{~m} / \mathrm{s}$ and external load of $5 \mathrm{M} \Omega$. The output power was larger than that of single-layer structure, and the power density was 1.3 $\mathrm{mW} / \mathrm{cm}^{3}$.

In 2011, Bryant et al. [21] proposed a wind energy generator with an airfoil hinged at the free end of PZT piezoelectric cantilever beam, as shown in Figure 16. The device vibrated to generate electricity through the flutter of the piezoelectric beam. When the size of the generator was $254 \mathrm{~mm} \times 25.4 \mathrm{~mm} \times 0.381$ $\mathrm{mm}$, and size of the piezoelectric vibrator was $46 \mathrm{~mm} \times 20.6 \mathrm{~mm} \times 0.254 \mathrm{~mm}$, the critical wind speed of the device was reduced to $1.9 \mathrm{~m} / \mathrm{s}$. When wind speed was $8 \mathrm{~m} / \mathrm{s}$, the output power was up to $2.2 \mathrm{~mW}$. However, the volume of the generator was larger because of the wing.

Figure 17 was a miniature generator that could collect multi-directional wind energy by Zhao in 2015 [22]. Unlike the traditional structure, the windward body of the generator adopted an arc-shaped flexible bell-like structure, and the PZT piezoelectric layer with a size of $20 \mathrm{~mm} \times 14 \mathrm{~mm} \times 0.2 \mathrm{~mm}$ was covered at the bottom of the arc-shaped flexible structure. Its critical starting wind speed was too low to $2 \mathrm{~m} / \mathrm{s}$. When the wind speed was up to $17 \mathrm{~m} / \mathrm{s}$ and the resistance was $15 \mathrm{k} \Omega$, the output power was $1.73 \mathrm{~mW}$. When the wind speed was $10.5 \mathrm{~m} / \mathrm{s}$, 18 LED lights in series were lit simultaneously. 


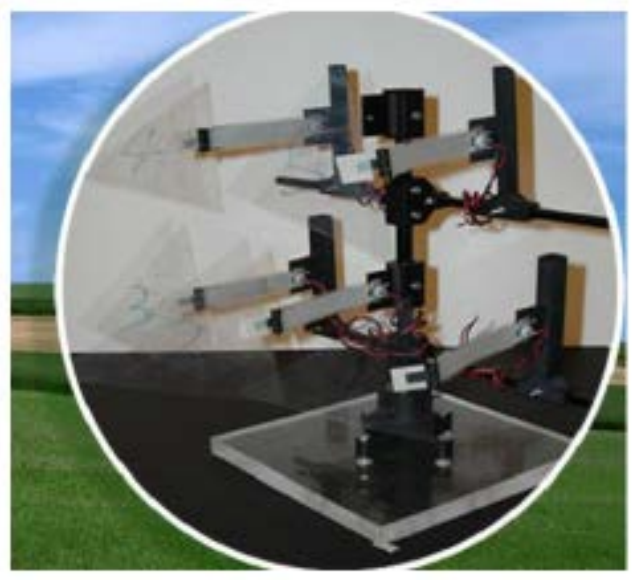

Figure 15. The piezoelectric bionic tree by Li [19].

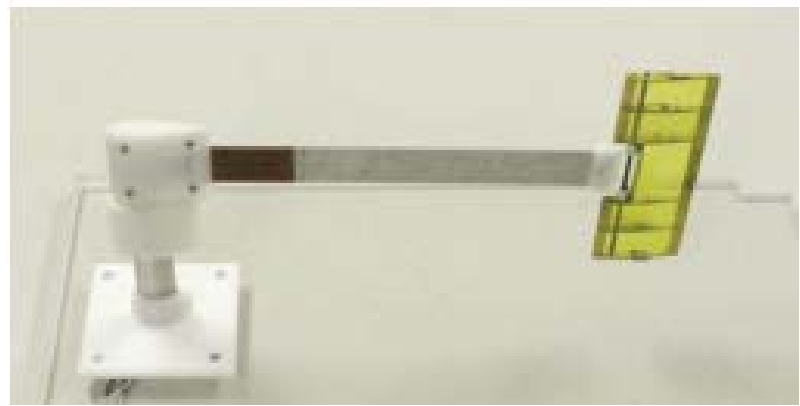

Figure 16. A wind energy generator with an airfoil by Bryant [21].

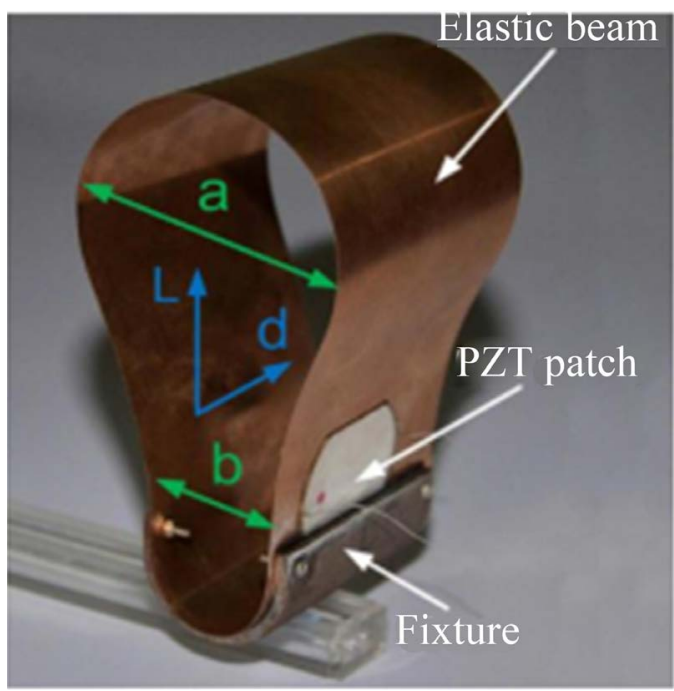

Figure 17. Bell-like piezoelectric generator by Zhao [22].

\subsection{Resonant Cavity Type}

This kind of piezoelectric generator has a resonant cavity which is used to change the flow field distribution and increase the starting load force acting on the piezoelectric oscillator for producing larger amplitude to generate more electricity. 
In 2011, IMEC of the European Centre for Microelectronics Research and Holst Center of the Netherlands jointly designed the kind of two generators, as shown in Figure 18 [23]. The first one in the left had a vacuum cylinder with a length of $17 \mathrm{~cm}$ and a diameter of $8 \mathrm{~cm}$. The length of the vent is $2 \mathrm{~cm}$, and the diameter of the hole is $2 \mathrm{~cm}$. The resonance frequency of this harvester was 160 $400 \mathrm{~Hz}$, it could work in the wind speed $10-15 \mathrm{~m} / \mathrm{s}$. The second resonator had a vacuum cylinder with length $3.5 \mathrm{~cm}$, diameter $6.2 \mathrm{~cm}$. The vent length was $1 \mathrm{~cm}$ and diameter $1.4 \mathrm{~cm}$. The resonant frequency of the generator was $553-900 \mathrm{~Hz}$, which could operate under the wind speed of $16-20 \mathrm{~m} / \mathrm{s}$. The piezoelectric vibrators in the two generators were MEMS cantilever structure. In order to improve the amplitude of self-excited vibration, latex film was installed at the bottom of the resonator. The test results showed that the output power of the first one was $2 \mathrm{uW}$ when the wind speed was $13 \mathrm{~m} / \mathrm{s}$, while the output power of the second was $42.2 \mathrm{uW}$ when the wind speed $20 \mathrm{~m} / \mathrm{s}$.

In 2010, Clair [24] proposed a resonant piezoelectric wind power generator by imitating the vibration principle of the reed when playing harmonica in Figure 19. The wind energy generator could generate $0.1-0.8 \mathrm{~mW}$ power in the range of $7.5-12.5 \mathrm{~m} / \mathrm{s}$ wind speed, and the maximum energy density reached 0.02 $\mathrm{mW} / \mathrm{cm}$.

In 2012, Du [25] proposed a piezoelectric wind energy generator with resonant cavity, as shown in Figure 20. The vibrator consisted of piezoelectric and flexible beams and was at a certain angle $\left(\alpha=12^{\circ}\right)$ from the cavity. The maximum power of $1.28 \mathrm{~mW}$ was generated when the overall size of the generator was $64 \mathrm{~mm} \times 22 \mathrm{~mm} \times 14 \mathrm{~mm}$, the length/width of the piezoelectric beam was $38 / 6.4 \mathrm{~mm}$, and the average wind speed $17 \mathrm{~m} / \mathrm{s}$. The device obviously improved the harvesting efficiency than the one without the flexible beam.

In 2014, Wang [26] proposed an electromagnetic wind energy generator with double bifurcated reed vibrator, as shown in Figure 21. The maximum output power and conversion efficiency reached $56 \mathrm{~mW}$ at $20.3 \mathrm{~m} / \mathrm{s}$ and $2.3 \%$ at $4 \mathrm{~m} / \mathrm{s}$ respectively when the size of the air inlet was $60 \mathrm{~mm} \times 60 \mathrm{~mm}$. The working wind speed range lied in $3.2-21.2 \mathrm{~m} / \mathrm{s}$. The device effectively increased the vibration amplitude of the reed and the effective working range of the wind speed.

In 2017, Zhang [27] proposed a miniature wind energy generator based on
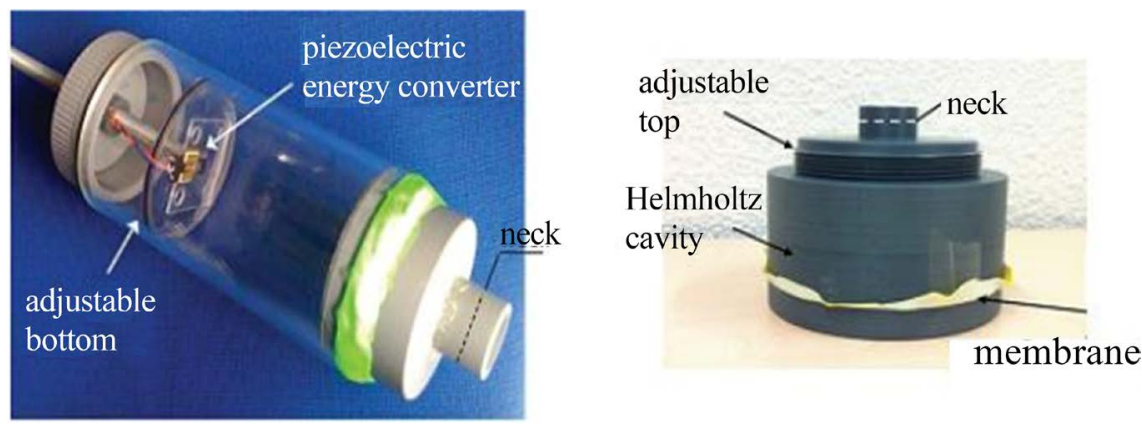

Figure 18. Two kinds of wind generators jointly designed by IMEC and Holst [23]. 


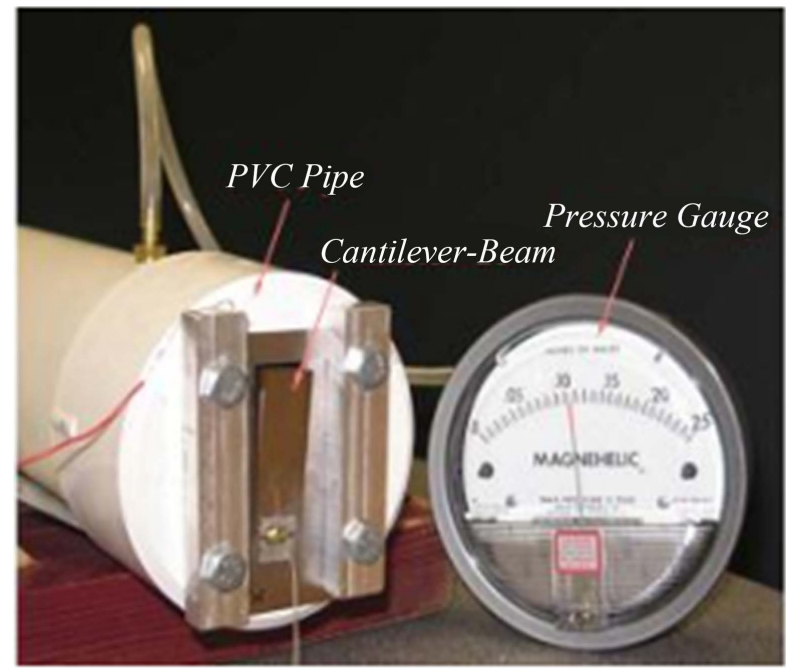

Figure 19. Piezoelectric wind energy generator with resonant cavity by Clair [24].

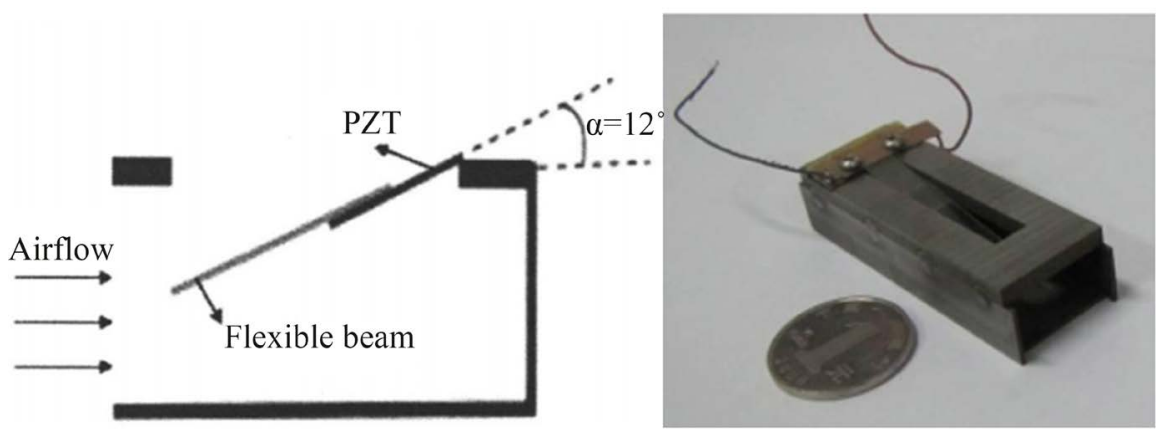

Figure 20. A generator with rigid and flexible beams by $\mathrm{Du}$ [25].

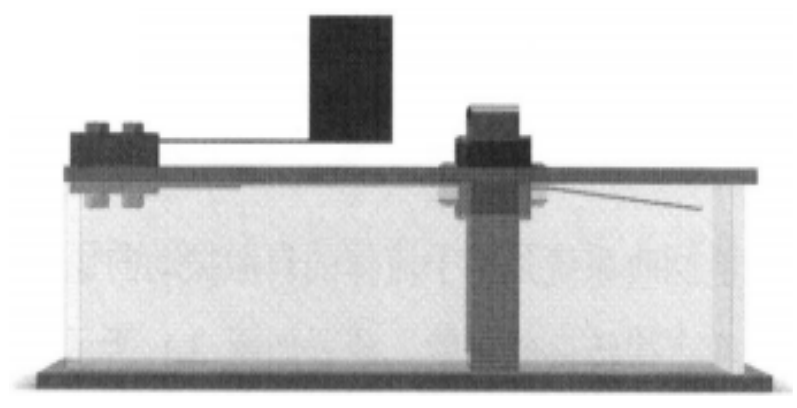

Figure 21. A generator with double bifurcated reed vibrator and magnet and resonant cavity by Wang [26].

tuning fork with double reed and resonant cavity, as shown in Figure 22. The structure had better harvesting performance from wind than the reed. When the cavity size was $40 \mathrm{~mm} \times 40 \mathrm{~mm} \times 140 \mathrm{~mm}$, the wind speed of $6 \mathrm{~m} / \mathrm{s}$ and the optimal load of $0.15 \mathrm{M} \Omega$, the output power was $0.83 \mathrm{~mW}$ and the energy conversion efficiency reached $0.62 \%$.

\subsection{Hybrid Type}

In order to further improve energy conversion efficiency, Che [28] designed a 


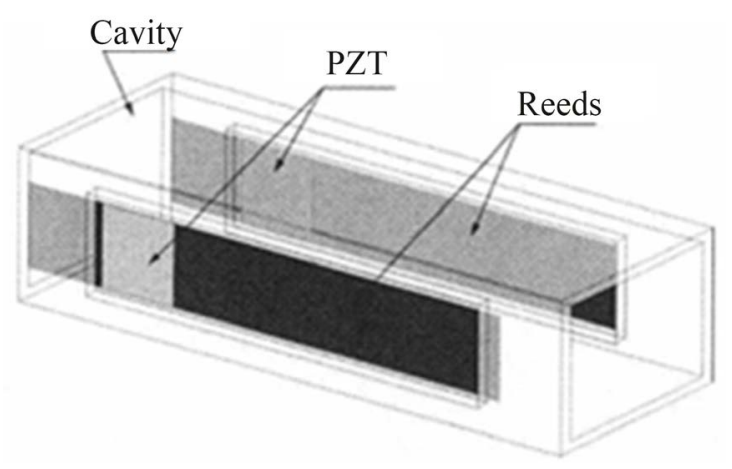

Figure 22. A generator based on tuning fork with double reed and resonant cavity by Zhang [27].

two-degree-of-freedom wind energy generator which combines galloping motion and vortex-induced vibration in 2014. Its working principle was shown in Figure 23. When the wind speed reached a critical starting value, the bluff body moved along left and right to drive two external beams to vibrate, and thus causing the piezoelectric vibrator deformation to generate electric energy. Meanwhile, the eddy current was formed thereafter the bluff body to force the middle beam to vibrate and generate electric energy. When the wind speed reached $6 \mathrm{~m} / \mathrm{s}$, the output power was $1.5 \mathrm{~mW}$.

In the same year, Zhao [29] designed a generator similar to that above one. The difference was that the permanent magnet was used as a mass at the free end of the inner beam and another magnet was installed at the corresponding position of the bluff body, as shown in Figure 24. Combined with magnetic force, the generator further reduced the critical wind speed to $1 \mathrm{~m} / \mathrm{s}$ and increased the output power. $4 \mathrm{~mW}$ electricity was captured at $5 \mathrm{~m} / \mathrm{s}$ wind speed. However, when the wind speed exceeded $4.5 \mathrm{~m} / \mathrm{s}$, the energy harvesting efficiency was not as good as the traditional galloping generator with one-degree-of-freedom.

In 2015, Cao [30] proposed a wind energy generator for TPMS power supply, as shown in Figure 25. The generator mixed the mechanism of direct excitation with resonant cavity and vortex-induced. It was integrated with the TPMS transmitter and installed in the valve core. It rotated along with the tire to supply power for the TPMS system. The harvesting energy part of the device was $\mathrm{T}$-shaped cantilever beam and extended to the outside of the cavity for effectively capturing periodic wind energy. In addition, the two sides of the cavity were opened and a cylinder was placed in the middle to generate vortex-induced vibration and disturb the airflow in the cavity for promoting the vibration of the piezoelectric beam. The experimental results showed that if the transverse part of the beam was $30 \mathrm{~mm} \times 10 \mathrm{~mm} \times 0.2 \mathrm{~mm}$ and the end transverse rectangle was $26 \mathrm{~mm} \times 16 \mathrm{~mm} \times 2.2 \mathrm{~mm}$, the system worked in the optimal working condition. The slender cylindrical structure with a diameter of $4 \mathrm{~mm}$ could improve the vibration of the beam. When the load was about $3 \mathrm{M} \Omega$, the maximum output power was about $0.0231 \mathrm{~mW}$, which could meet the requirement of TPMS system. 


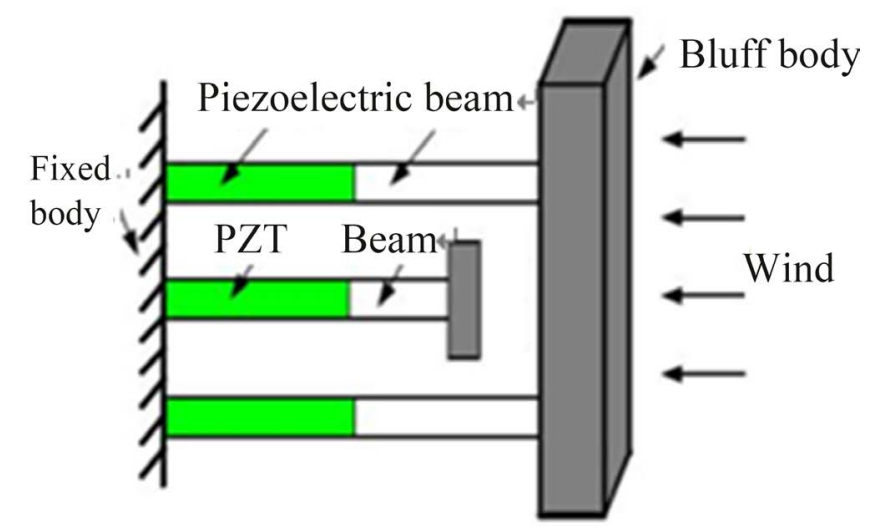

Figure 23. Two-degree-of-freedom wind energy generator by Che [28].

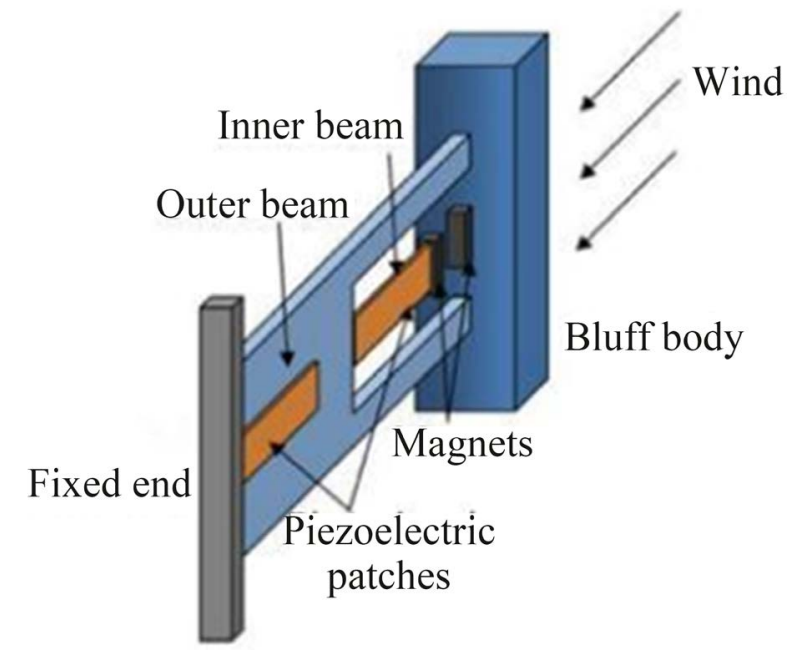

Figure 24. Two-degree-of-freedom wind energy generator with magnet by Zhao [29].

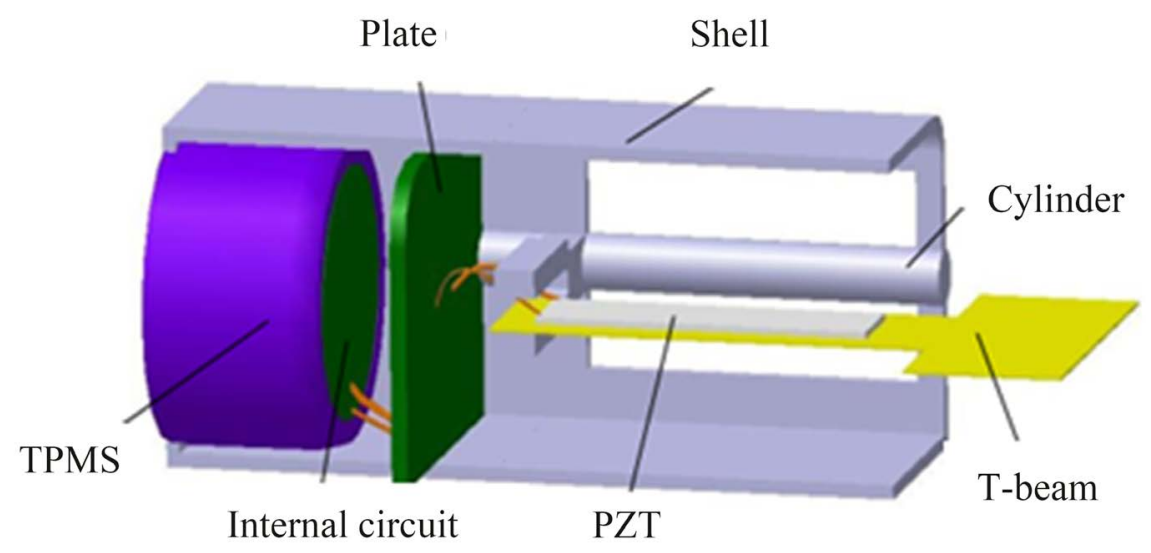

Figure 25. Hybrid wind energy generator for TPMS by Cao [30].

In 2018, Shu [31] proposed a hybrid structure for adding a bluff body in the resonant cavity. The results showed that the output power of the generator was larger than one of the collector without resonant cavity in the range of $2-12 \mathrm{~m} / \mathrm{s}$ wind speed. 


\section{Comparison of Piezoelectric Materials and Piezoelectric Vibrators}

The choice of piezoelectric materials and piezoelectric vibrators is important for energy conversion efficiency and service life of piezoelectric generators. The selection of piezoelectric materials should take into consideration the working environment and material properties. Commonly used piezoelectric materials in wind energy piezoelectric generator include PZT piezoelectric ceramics and PVDF polyvinylidene fluoride thin films. The properties of two materials are shown in Table 1.

It can be derived from the Table that PZT is widely used in wind energy piezoelectric generators for its larger piezoelectric constants, dielectric constants and electromechanical coupling coefficients. It has excellent performance and can obtain high voltage with small deformation. However, it is easy for PZT to happen fatigue fracture under long-term vibration because of its brittle. Meanwhile, it is difficult to deform under general wind force for its large stiffness. These shortcomings become more seriously under micro-device design.

In contrast, PVDF is a soft plastic film that can be made of $0.5 \mathrm{~mm}-2 \mathrm{~mm}$ thickness. It has the advantages of light weight, small distortion, good flexibility, high stability, suitable mechanical strength. It is not easy for PVDF to break and fatigue and more suitable for designing micro wind energy piezoelectric vibrators, especially composite surface piezoelectric ones [33] [34] [35].

Either PZT or PVDF was used, all piezoelectric vibrators adopted cantilever beam structure because the cantilever is simpler and easier to produce vibration deformation and generate electric energy under the action of breeze or external force than other ones.

\section{Characteristics of Various wind Energy Piezoelectric Generators}

In summary, windmill piezoelectric generators generally contain many parts such as wind blades and turbines. The process of manufacturing and assembling is more complex. When the dialer, convex teeth and steel ball are selected as the exciting parts, there exists not only serious friction loss but also noise among the exciting parts and piezoelectric oscillator and rotating body. The working needs a larger starting moment. In particular, the smaller the size of the generator, the more complex the processing and assembly of parts are, the greater the proportion of friction loss and the worse the wear of components are. When magnets

Table 1. Properties of PZT and PVDF [32].

\begin{tabular}{ccccl}
\hline Materials & $\mathrm{d}_{31}(\mathrm{pm} / \mathrm{V})$ & $\mathrm{e}_{31}\left(\mathrm{C} \cdot \mathrm{m}^{2}\right)$ & $\mathrm{K}_{31}$ & \multicolumn{1}{c}{ Properties' comparison } \\
\hline PZT & 175 & 6.5 & $0.34 \begin{array}{l}\text { Large piezoelectric coefficient, brittleness and } \\
\text { density }\end{array}$ \\
PVDF & 28 & 0.06 & $0.12 \begin{array}{l}\text { Large flexibility, light weight and large mechanical } \\
\text { impedance }\end{array}$ \\
\hline
\end{tabular}


are used to form contactless excitation, although the efficiency and adaptability of power generation are improved to a certain extent and the starting moment is reduced, friction loss cannot be avoided between rotating parts, the volume of power generation device cannot decrease a lot. In addition, there is only one excitation frequency to match the natural frequency of windmill generator, so the effective working frequency range is narrow.

The three types of vortex-induced, galloping motion and resonant cavity contain bluff body or resonant cavity, which makes the size of wind energy generators relatively larger in volume and more complex. So it is not suitable for low wind speed occasions or for miniaturization.

The hybrid type of wind energy piezoelectric generator adopts two or more mechanisms to collect low frequency and multi-directional wind. So it has characteristics of broadband and high conversion efficiency. Although designing this kind of generator is more complex, it is easy to achieve integration and miniaturization if the appropriate mixing mode and structure are chosen.

The characteristics and applicability of different types of wind energy piezoelectric generators are shown in Table 2.

\section{Future Trends}

With the continuous improvement of integrated circuit technology and micro/ nano electromechanical system technology, micro-devices have become an inevitable trend in the development of micro electromechanical systems. The volume of micro-devices will be smaller and smaller, and the demand for energy will be less and less. As shown in Table 3, the power consumption of sensors in

Table 2. Characteristics and applicability of different types of wind energy piezoelectric generators.

\begin{tabular}{|c|c|}
\hline Excitation mode & Characteristics and applicability \\
\hline Windmill type & $\begin{array}{l}\text { - To collect multi-directions winds } \\
\text { - More components, complex structure } \\
\text { - Easily wear parts } \\
\text { - Large energy loss and low conversion efficiency } \\
\text { - High starting speed and not suitable for low wind speed environment } \\
\text { - Narrowly effective wind speed } \\
\text { - Narrow working band } \\
\text { - Low working stability } \\
\text { - Difficult to integrate and miniaturize }\end{array}$ \\
\hline $\begin{array}{l}\text { Vortex-induced and } \\
\text { galloping motion types }\end{array}$ & $\begin{array}{l}\text { - Moderate parts } \\
\text { - Unidirectional wind } \\
\text { - Unsuitable for low wind speed } \\
\text { - Narrow working band } \cdot \text { Moderate conversion efficiency } \\
\text { - Easy integration and miniaturization }\end{array}$ \\
\hline Resonant cavity type & $\begin{array}{l}\text { - More complicated structure } \\
\text { - Unidirectional wind } \\
\text { - Relatively wide working band } \\
\text { - Low starting wind speed } \cdot \text { Moderate conversion efficiency } \\
\text { - Difficulty to integrate and miniaturize }\end{array}$ \\
\hline
\end{tabular}


Continued

\begin{tabular}{ll}
\hline & - simple structure and small the volume \\
- Suitable for low wind speed \\
Directly excited type Relative wide working band \\
- Higher conversion efficiency \\
- Easy integration and miniaturization \\
- More complicated structure \\
- To collect multi-directions winds \\
- Wind working band. Low starting speed \\
- High conversion efficiency \\
- Easy integration and miniaturization
\end{tabular}

Table 3. Power consumption of commonly used sensors in network [37].

\begin{tabular}{cccccc}
\hline $\begin{array}{c}\text { Sensor } \\
\text { types }\end{array}$ & $\begin{array}{c}\text { Pressure } \\
\text { sensor }\end{array}$ & $\begin{array}{c}\text { Temperature } \\
\text { sensor }\end{array}$ & $\begin{array}{c}\text { Humidity } \\
\text { sensor }\end{array}$ & $\begin{array}{c}\text { Acoustic } \\
\text { sensor }\end{array}$ & $\begin{array}{c}\mathrm{RF} \\
\text { transmitter }\end{array}$ \\
\hline $\begin{array}{c}\text { Power } \\
\text { consumption (mW) }\end{array}$ & $2.2-3.6$ & $2.5-5.5$ & $1.3-3$ & $1.5-5$ & $0.3-3$ \\
\hline
\end{tabular}

network has been reduced to less than $10 \mathrm{~mW}$. With the development of microelectronics technology, the power consumption is lower and lower. That makes possible to realize self-supply of micro-devices using the wind energy piezoelectric generators.

Wind energy piezoelectric generator is an effective choice to achieve selfpowered for micro-devices. In recent years, scholars have done a lot of research on various wind energy piezoelectric micro-generators and have achieved fruitful results. It has been successfully applied to wireless sensor nodes [23] [36], wireless wind speed monitoring node [37], TPMS [30] and so on. However, many wind piezoelectric generators still have some disadvantages such as large volume and high starting wind speed, which make it difficult to be directly applied to self-powered micro-devices. Summarizing the current research status of wind energy piezoelectric generator, a lot of work still needs to be done to realize the miniaturization of wind energy piezoelectric generator for meeting selfpowered micro-devices. Therefore, it is necessary to innovate design concepts, develop new materials and use new methods to research in depth towards the goal of miniaturization, lightweight and integration.

1) Development of new piezoelectric materials. At present, only two piezoelectric materials are commonly applied: PZT and PVDF. Ideal piezoelectric materials with high electromechanical coupling coefficient and good flexibility can significantly improve the power generation performance of piezoelectric power generation devices and adapt to complex surface configuration, which is also very helpful to the planning and design of new configuration of piezoelectric oscillators. Therefore, the application and development of new piezoelectric materials should be further strengthened.

2) Research on broadband and multi-directional energy harvesting technology. Due to the instability and diversity of wind sources in the environment, the 
conversion efficiency of a single fundamental frequency piezoelectric oscillator is not high. Therefore, it is necessary to carry out research on broadband and multi-directional energy harvesting technology. The technology will make piezoelectric vibrator resonate or approximate resonant in a certain wide frequency band to improve its conversion efficiency and environmental adaptability and power generation efficiency.

3) Exploring multi-mode coupling mechanism. The wind energy piezoelectric generator based on single mechanism has low energy conversion efficiency and is difficult to adapt for complex and changeable environment. The adoption of multi-mode coupling is an alternative way to meet the needs of future technological development. Therefore, the research of multi-mode coupling mechanism and the development of multi-mode piezoelectric vibrators is the key.

4) Development of micro integrated system. Although the main characteristics of piezoelectric harvesting energy are particularly suitable for the supply of micro-electromechanical systems, advanced design concepts and manufacturing technologies must be adopted to achieve miniaturization, lightweight and further integration with micro-devices. By using the technology of CAD/CAM/CAM and fusion design, the overall structure design and optimization are carried out. Meanwhile, by breaking through the limitation of traditional material selection, light materials such as nylon and PET are selected to improve the flexibility of the system while enhancing the strength and reducing the weight. 3D printing technology and thick film screen printing technology are used to carry out the whole one-time processing of the micro-devices.

\section{Conflicts of Interest}

The authors declare no conflicts of interest regarding the publication of this paper.

\section{References}

[1] Vullers, R.J.M., Schaijk, R.V., Visser, H.J., et al. (2010) Energy Harvesting for Autonomous Wireless Sensor Networks. IEEE of Solid-State Circuits Magazine, 2, 29-38. https://doi.org/10.1109/MSSC.2010.936667

[2] Harb, A. (2011) Energy Harvesting: State-of-the-Art. Renewable Energy, 36, 26412654. https://doi.org/10.1016/j.renene.2010.06.014

[3] Priya, S., Chen, C.T., Fye, D., et al. (2005) Piezoelectric Windmill: A Novel Solution to Remote Sensing. Japanese Journal of Applied Physics, 44, L104.

[4] Myers, R., Vickers, M., Kim, H., et al. (2007) Small Scale Windmill. Applied Physics Letters, 90, 1-3. https://doi.org/10.1063/1.2435346

[5] Zhang, C. (2009) Theoretical Research and Innovative Design of Wind Energy Conversion Device Based on Piezoelectric Materials. Xi'an Science and Technology University, Xi'an.

[6] Yang, Y., Shen, Q., Jin, J., et al. (2014) Rotational Piezoelectric Wind Energy Harvesting Using Impact-Induced Resonance. Applied Physics Letters, 105, 053901. https://doi.org/10.1063/1.4887481

[7] Song, G.L. (2016) Research on PVDF Piezoelectric Wind Energy Collection System 
Based on Impact Vibration. Dalian University of Technology, Dalian.

[8] Xu, G.J. (2017) Research on Piezoelectric Harvesting Technology from Environment. Nanjing University of Information Science and Technology, Nanjing.

[9] Luo, Z.S. (2018) Design and Research of a New Piezoelectric Wind Energy Collector. Electronic University of Science \& Technology of Hangzhou, Hangzhou.

[10] Karami, M.A., Farmer, J.R. and Inlllan, D.J. (2013) Parametrically Exceed Nonlinear Piezoelectric Compact Wind Turbine. Renewable Energy, 50, 977-987. https://doi.org/10.1016/j.renene.2012.07.037

[11] Rezaei-Hosseinabadi, N., Tabesh, A. and Dehghani, R. (2015) A Topology and Design Optimization Method for Wideband Piezoelectric Wind Energy Harvesters. IEEE Transactions on Industrial Electronics, 63, 2165-2173.

[12] Ji, X. (2016) Design of a Wide-Band Rotary Piezoelectric Generator Driven by a Breeze. Dalian University of Technology, Dalian.

[13] Akaydin, H.D., Elvin, N. and Reopoulos, Y. (2010) Energy Harvesting from Highly Unsteady Fluid Flows Using Piezoelectric Materials. Journal of Intelligent Material Systems and Structures, 21, 1263-1278. https://doi.org/10.1177/1045389X10366317

[14] Weinstein, L.A., Cacan, M.R., So, P.M., et al. (2012) Vortex Shedding Induced Energy Harvesting from Piezoelectric Materials in Heating, Ventilation and Air Conditioning Flows. Smart Materials and Structures, 21, 045003. https://doi.org/10.1088/0964-1726/21/4/045003

[15] Sun, H., Zhu, D., White, N.M., et al. (2013) A Miniature Airflow Energy Harvester from Piezoelectric Materials. Journal of Physics. Conference Series, IOP Publishing, 012057.

[16] He, X.F. and Gao, J. (2013) Wind Energy Harvesting Based on Flow-Induced-Vibration and Impact. Microelectronic Engineering, 111, 82-86. https://doi.org/10.1016/j.mee.2013.02.009

[17] Sirohi, J. and Mahadik, R. (2011) Piezoelectric Wind Energy Harvester for LowPower Sensors. Journal of Intelligent Material Systems and Structures, 22, 2215 2228. https://doi.org/10.1177/1045389X11428366

[18] Hu, G., Tse, K.T. and Kwok, K.C.S. (2016) Enhanced Performance of Wind Energy Harvester by Aerodynamic Treatment of a Square Prism. Applied Physics Letters, 108, Article ID: 123901. https://doi.org/10.1063/1.4944555

[19] Li, S.G. and Lipson, H. (2009) Vertical-Stalk Flapping-Leaf Generator for Wind Energy Harvesting. Proceedings of the ASME Conference on Smart Materials, Adaptive Structures and Intelligent Systems, 611-619. https://doi.org/10.1115/SMASIS2009-1276

[20] Li, S., Yuan, J. and Lipson, H. (2011) Ambient Wind Energy Harvesting Using Cross-Flow Fluttering. Journal of Applied Physics, 109, Article ID: 026104. https://doi.org/10.1063/1.3525045

[21] Bryant, M. and Garcia, E. (2011) Modeling and Testing of a Novel Aeroelastic Flutter Energy Harvester. Journal of Vibration and Acoustics, 133, Article ID: 011010. https://doi.org/10.1115/1.4002788

[22] Zhao, J., Yang, J., Lin, Z., et al. (2015) An Arc-Shaped Piezoelectric Generator for Multi-Directional Wind Energy Harvesting. Sensors and Actuators a Physical, 236, 173-179. https://doi.org/10.1016/j.sna.2015.10.047

[23] Matova, S.P., Elfrink, R., Vullers, R.J.M., et al. (2011) Harvesting Energy from Airflow with a Michromachined Piezoelectric Harvester inside a Helmholtz Resonator. Journal of Micromechanics and Microengineering, 21, Article ID: 104001. 
https://doi.org/10.1088/0960-1317/21/10/104001

[24] Clair, D.S., Bibo, A., Sennake savababu, V.R., et al. (2010) A Scalable Concept for Micropower Generation Using Flow-Induced Self-Excited Oscillations. Applied Physics Letters, 96, Article ID: 144103. https://doi.org/10.1063/1.3385780

[25] Du, Z. and He, X. (2012) Miniature Piezoelectric Wind Energy Harvester with Resonant Cavity. Journal of Sensing Technology, 25, 748-750.

[26] Wang, X. (2014) Electromagnetic Wind Energy Generation Device Based on Double Bifurcated Reed and Resonant Cavity Structure. Suzhou University, Suzhou.

[27] Zhang, Y. (2017) Research on Wind Energy Recovery Device of Tuning Fork Reed Piezoelectric Resonator. Hefei Polytechnic University, Hefei.

[28] Che, X. (2014) Design and Research of Miniature Piezoelectric wind Energy Harvestor. Xi'an Electronic and Science University, Xian.

[29] Zhao, L., Tang, L. and Yang, Y. (2014) Enhanced Piezoelectric Galloping Energy Harvesting Using 2 Degree-of-Freedom Cut-Out Cantilever with Magnetic Interaction. Japanese Journal of Applied Physics, 53. https://doi.org/10.7567/JJAP.53.060302

[30] Cao, Y. (2015) Research on Wind-Induced Vibration Energy Harvesting System Applied to TPMS. Nanjing University of Aeronautics \& Astronautics, Nanjing.

[31] Shu, C., Zhang, J., Wu, S., et al. (2018) A Piezoelectric Wind Energy Collector with Resonant Cavity. Journal of Vibration and Shock, 37, 22-26.

[32] Anton, S.R. and Sodano, H.A. (2007) A Review of Power Harvesting Using Piezoelectric Materials (2003-2006). Smart Materials and Structures, 16, R1-R21. https://doi.org/10.1088/0964-1726/16/3/R01

[33] Tang, G. (2013) Research on MEMS Vibration Energy Harvester Based on Piezoelectric Thick Film. Shanghai Jiao Tong University, Shanghai.

[34] Lee, C.S., Joo, J., Han, S., Lee, J.H. and Koh, S.K. (2005) Poly (Vinylidene Fluoride) Transducers with Highly Conducting Poly (3,4-Ethylenedioxythiophene) Electrodes. Synthetic Metals, 152, 49-52. https://doi.org/10.1016/j.synthmet.2005.07.116

[35] Vatansever, D., Hadimani, R.L., Shah, T., et al. (2011) An Investigation of Energy Harvesting from Renewable Sources with PVDF and PZT. Smart Materials and Structures, 20, Article ID: 055019. https://doi.org/10.1088/0964-1726/20/5/055019

[36] Huang, N. (2014) Wind-Induced Vibration Piezoelectric Energy Harvesting Technology for Stress Sensor Nodes of Hoist Cylinder. China University of Mining and Technology, Xuzhou.

[37] Chen, Y. (2014) Wireless Wind Speed Monitoring Node Based on Micro Piezoelectric Energy Harvester. Chongqing University, Chongqing. 